\title{
RESÍDUOS SÓLIDOS DE MADEIRA: APLICABILIDADE DE RESÍDUO DE SERRAGEM DE MDF NO DESIGN DE AMBIENTES
}

\author{
Laís Soares Diniz Pinto \\ Universidade Federal de Minas Gerais \\ lais.sodinipi@gmail.com \\ Cynthia Casagrande Matos \\ Universidade do Estado de Minas Gerais \\ cynthiacasagrande@gmail.com \\ Márcia Luiza França da Silva \\ Universidade Federal de Minas Gerais \\ marcial@arq.ufmg.br
}

Resumo: A reutilização de resíduos de MDF (Medium Density Fiberboard) com resinas poliméricas naturais seja ela vegetal ou animal, não sintéticas, podem conter propriedade mecânicas e físicas consideráveis enquanto material de construção para novos produtos. Com base na pesquisa de campo referente ao contexto das indústrias moveleiras e identificação dos tipos de resíduos produzidos principalmente pelas fábricas situadas na região de Belo Horizonte, percebe-se o potencial limitado da redução dos impactos ambientais causados pelas indústrias moveleiras. $O$ setor moveleiro, durante e após o ciclo produtivo, apresenta um relevante desperdício desses resíduos sólidos de MDF, o que torna um fator preocupante para o meio ambiente, além da falta de incentivo em pesquisas e investimento em inovações tecnológicas para aproveitamento desse material descartado. O objetivo deste projeto é a partir das informações coletadas, estudar o aproveitamento dos resíduos de MDF, afastando a ideia de resíduo, lixo ou de material rejeitado pelas indústrias que por sua vez não consideram como parte integrante do seu processo. Neste contexto, o design, servirá como ferramenta principal, para encontrar soluções, buscando a valorização estética desse novo produto e conferir um significado de apelo comercial para o consumo do mercado da decoração e arquitetura.

Palavras-chave: Setor Moveleiro; Resíduo de MDF; Revestimento de Parede; Design; Aglutinantes.

\footnotetext{
Abstract: The reuse of waste MDF (Medium Density Fiberboard) with natural polymeric resins, whether plant or animal, non-synthetic, may contain considerable mechanical and physical properties as a construction material for new products. Based on field research in the context of the furniture industry and identify the types of waste mainly produced by factories located in the
} 
region of Belo Horizonte, one sees limited potential for reducing environmental impacts caused by the furniture industry. For the furniture sector during and after the production cycle presents a significant waste of these solid waste MDF, which makes it a concern for the environment, and the lack of incentive for research and investment in technological innovations for use of this discarded material. The objective of this project is to generate from the information collected seek opportunities and feasible proposals for the recovery of waste MDF, pushing the idea of waste, garbage or materials rejected by industry which in turn do not consider as part of your process. In this context, the design will serve as the main tool to find solutions, seeking the aesthetic value of this new product and give a meaning of commercial appeal for the consumption of the decoration and architecture market.

Keywords: Furniture sector; MDF residue; wall coverings; design; binders.

\section{INTRODUÇÃO}

Atualmente, muitos projetos utilizando resíduos de madeira, assim como também a serragem e recursos para concepção de novos produtos, conduzem a avaliar e considerar novas formas de consumo, despertando nas empresas e nos consumidores uma nova postura com relação aos impactos ambientais e suas consequências, direcionando muitos a assumir novos compromissos ligados a uma consciência ambiental e a preservação dos recursos naturais importantes para existência dos seres vivos.

Dessa forma, apropriar-se do uso desses recursos oriundos de matérias primas nobres em grande quantidade, discriminados na pesquisa através das observações feitas nos locais das visitas, registros fotográficos e por meio do levantamento de dados através de informações coletadas de outros projetos e pesquisas referentes ao aproveitamento dos resíduos de madeira, leva a viabilizar um produto com potencial mercadológico através das ferramentas do design. Visando aumentar a vida útil dos resíduos de $\mathrm{MDF}^{1}$ usados em larga escala pelas indústrias dos setores moveleiros, o reaproveitamento desse material considera a possibilidade por meio da reciclagem como fator de reutilização da matéria - prima residual contribuindo e respeitando esses recursos naturais que a natureza leva anos para gerar. Uma das contribuições do design é permitir que o resíduo deixasse de assumir um significado negativo para empresas e consumidores e se torne um produto com valor econômico, mas também devido o seu valor atrativo de responsabilidade ambiental e de incentivo a pesquisa e novas tecnologias (NAHUZ, 2005; TEIXEIRA \& CÉSAR, 2006; RODRIGUES, 2010).

\footnotetext{
${ }^{1}$ MDF é a sigla de Medium Density Fiberboard, é um material derivado da madeira e é internacionalmente conhecido por MDF. Em português a designação correta é placa de fibra de madeira de média densidade. fabricado através da aglutinação de fibras de madeira com resinas sintéticas e outros aditivos. O material é moldado em painéis lisos sob alta temperatura e pressão.
} 
O polo moveleiro é um dos principais consumidores de madeira, sendo assim, é possível perceber a importância do reaproveitamento dos resíduos gerados e a necessidade de criar a consciência de minimizar os seus impactos após o descarte. De acordo com a pesquisa realizada durante o ano de 2004, como ilustrado na Figura 1, é possível perceber que $45 \%$ dos resíduos são destinados para queima sem uma utilização adequada. Além disso, cerca de $6 \%$ são abandonados. Outros $24 \%$ são utilizados para produzir carvão. O restante é utilizado em fornos de olarias (5\%), usado para gerar energia elétrica (5\%), ou outros usos diversos (15\%) (SOUZA \& MARTINS MARCOS, 2008).

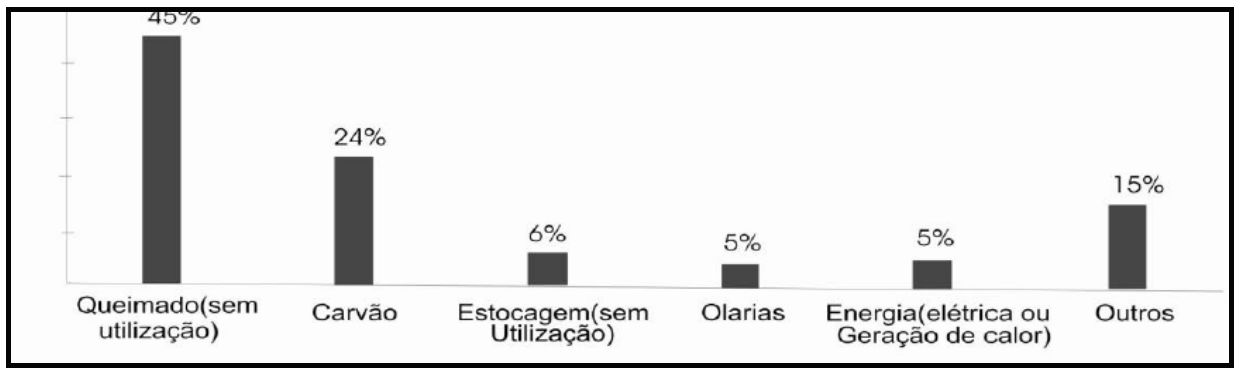

Figura 1: Principais usos dos resíduos de madeira em empresas madeireiras. onte: IMAZON, 2004.

Através da análise do ciclo de vida demonstrado através da Figura 2, percebe-se que desde a extração da madeira até o destino final dos resíduos de madeira oriundos de processos produtivos diversos problemas de descarte inadequado e sem um gerenciamento e acompanhamento das empresas geradoras. O descarte da madeira ainda é uma questão pouco considerada nos processos envolvendo o uso da madeira para projetos de móveis (CARVALHO, 2000; OLIVEIRA, 2009).

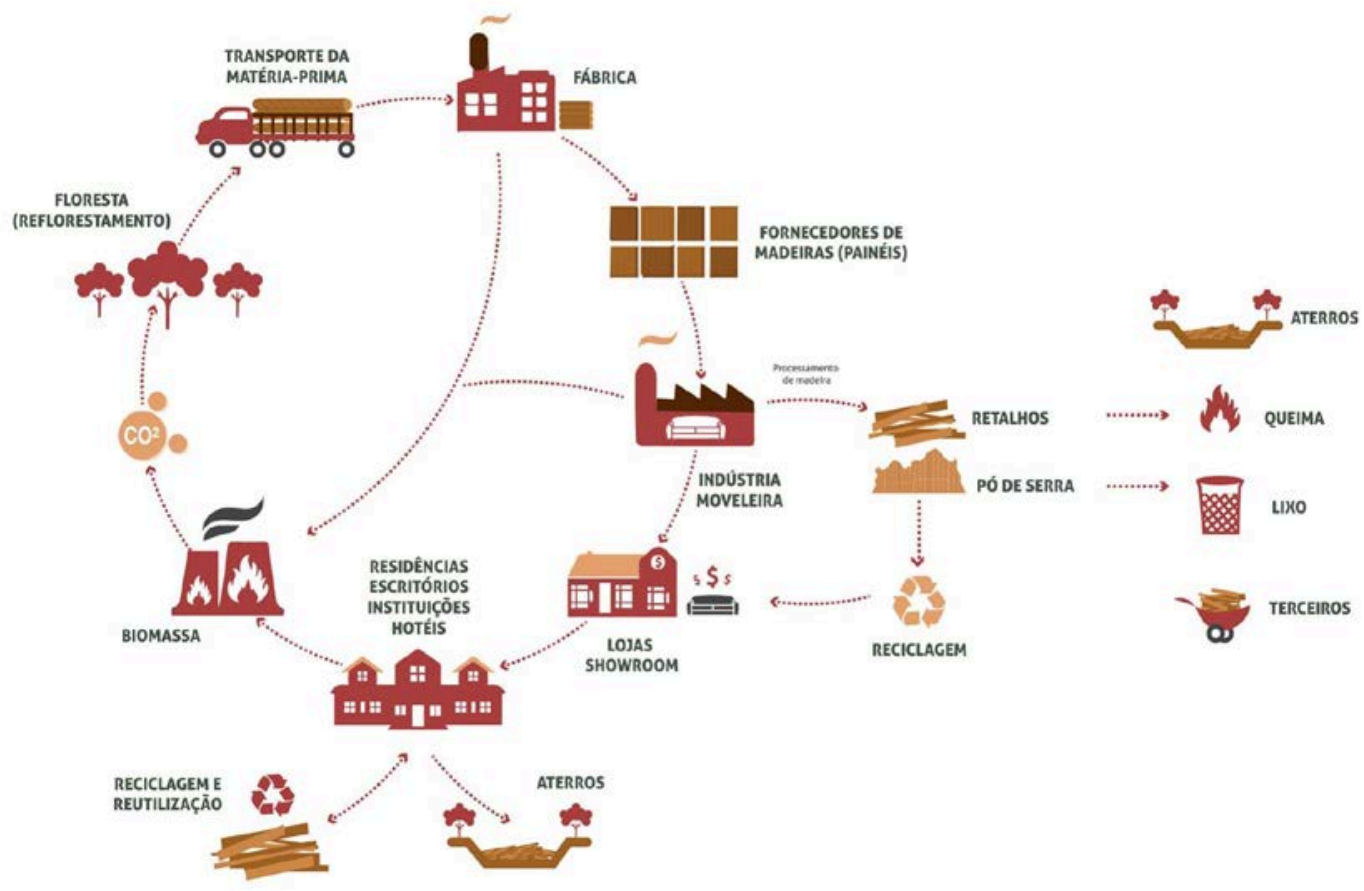

Figura 2: Análise do ciclo de vida.

Elaborado pelo autor, com base na pesquisa realizada, 2015. 
$\mathrm{Na}$ análise da cadeia produtiva acima envolvendo painéis de madeira, principalmente chapas de MDF, observam-se diversos impactos sobre o ambiente e, dentre esses impactos, um dos mais preocupantes hoje, é os resíduos gerados dos processos produtivos das indústrias moveleiras. Sendo, muito deles depositados em aterros ou para queimas a céu aberto. Um fator preocupante, pois, as chapas de aglomerados através da queima ocasiona a liberação de compostos químicos agregados à madeira e seus derivados, como o uso de formaldeído, uma substância tóxica e cancerígena, sendo prejudicial a saúde humana e também para contaminação dos solos. Uma das formas de solucionar o problema é a reciclagem desse material, como alternativa de transformar resíduos sólidos de madeira, como matéria-prima para outros processos produtivos, tanto para geração de energia quanto para criação de novos produtos (BOM, 2008; TUOTO, 2009; CÉSAR, 2009; CUNHA et al. 2010; GRUPO SUSTENTAX, 2010).

Baseado na pesquisa de campo foi possível observar durante as visitas técnicas referentes aos resíduos gerados durante o processo produtivo que o resíduo das fábricas em sua maioria gera inúmeros problemas como:

i) Ocupação de espaço podendo gerar infestação de proliferação de insetos;

ii) Degradação do meio ambiente e mistura de resíduos, sem a sua separação adequada, além de serem descartados com outros tipos de "lixos" como registrado da Figura 3;
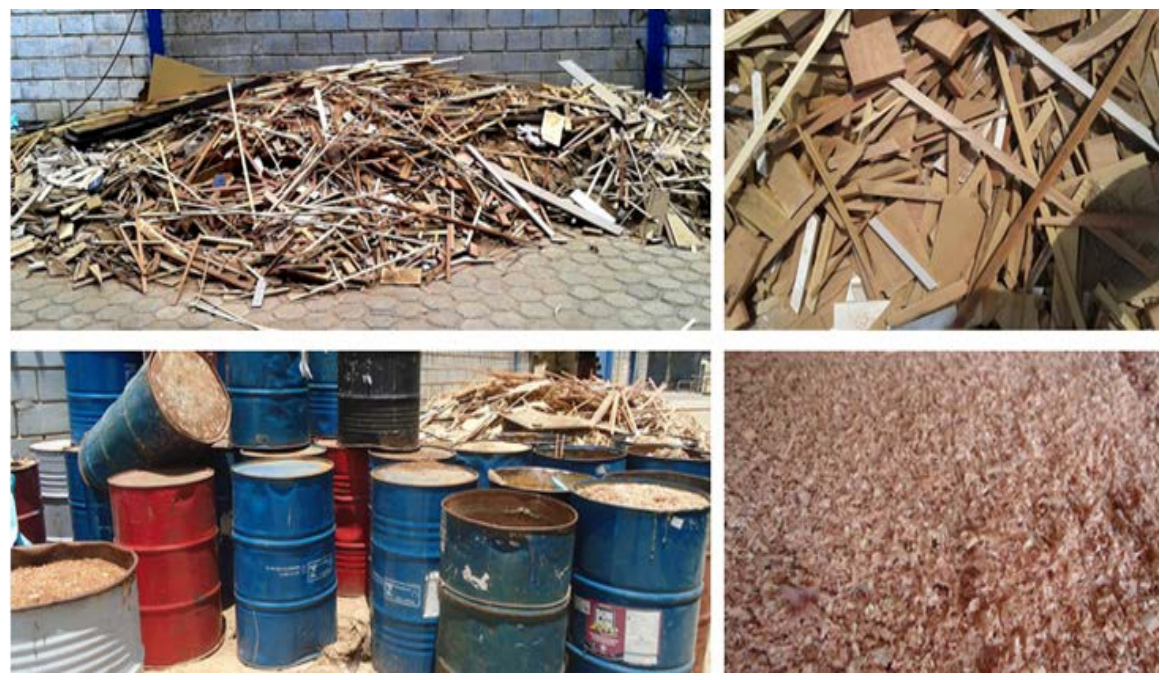

Figura 3: Acomodação dos resíduos de madeira, 2015.

Elaborado pelo autor, com base na pesquisa realizada.

iii) Riscos aos operários, podendo gerar acidente de trabalho sério, além do risco à saúde quanto ao descarte de forma indevida próxima a região da empresa, através da poluição dos solos, infestação de insetos (epidemia da dengue), e quando queimados em fornos ou a céu aberto com a liberação de substâncias tóxicas.

iv) Os resíduos são empilhados e acumulados, ocupando espaços nos pátios e galpões das fábricas como registrados na Figura 3. O grande problema desses 
empilhados de resíduos é a falta de controle por parte dos gerados, da quantidade de material descartado e da verificação do potencial do resíduo para aproveitamento. Normalmente os resíduos são removidos semanalmente e entregue para terceiros.

Os resíduos estudados foram registrados por meio de fotografias e para sua classificação foram levados em conta tamanhos, formas, colorações e acabamentos. Nos resíduos estudados e coletados em alguns casos os resíduos de MDF se encontravam misturados a outros tipos de madeiras, como: compensado, madeira maciça - eucalipto e punis, com painéis laminados e crus e de diversas espessuras. Os resíduos registrados no local foram classificados e em retalhos e serragem/pó de serra.

Retalhos: Separados e classificados de acordo com a coloração, forma, textura, espessura, tamanho, tipo de corte, peças laminadas ou cruas, volume e dimensões (Figura 4).

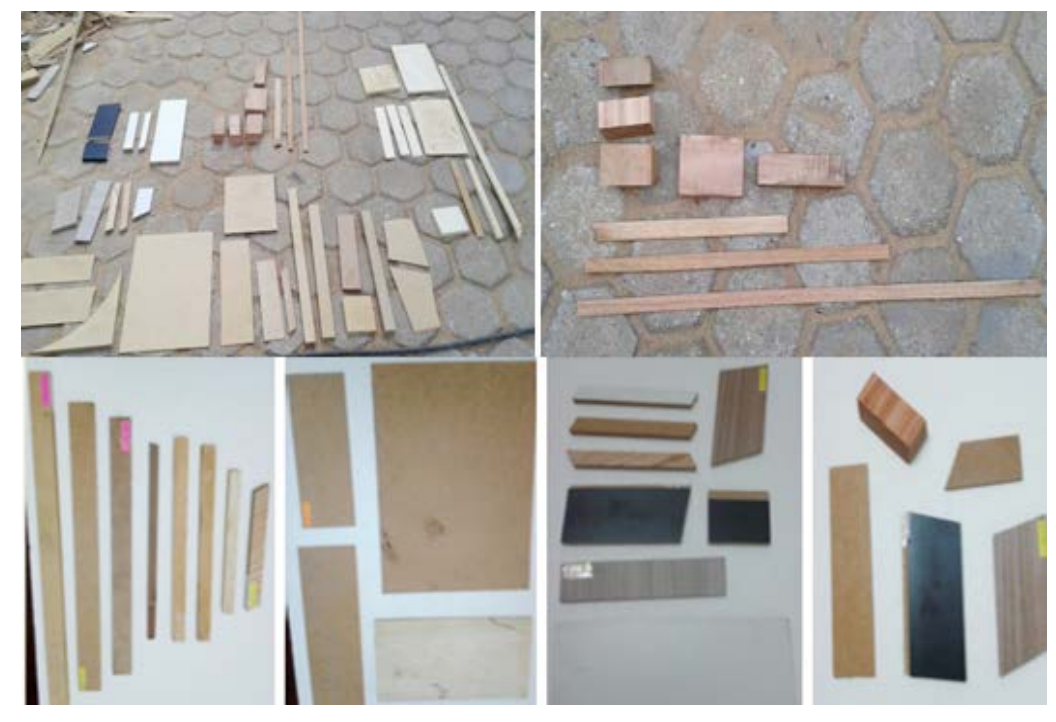

Figura 2: Catalogação dos retalhos de madeira, 2015.

Elaborado pelo autor, com base na pesquisa realizada.

Serragem/ Pó de serra: Separados e classificados de acordo com a coloração, granulometria, o tipo de processo e volume (Figura 5).

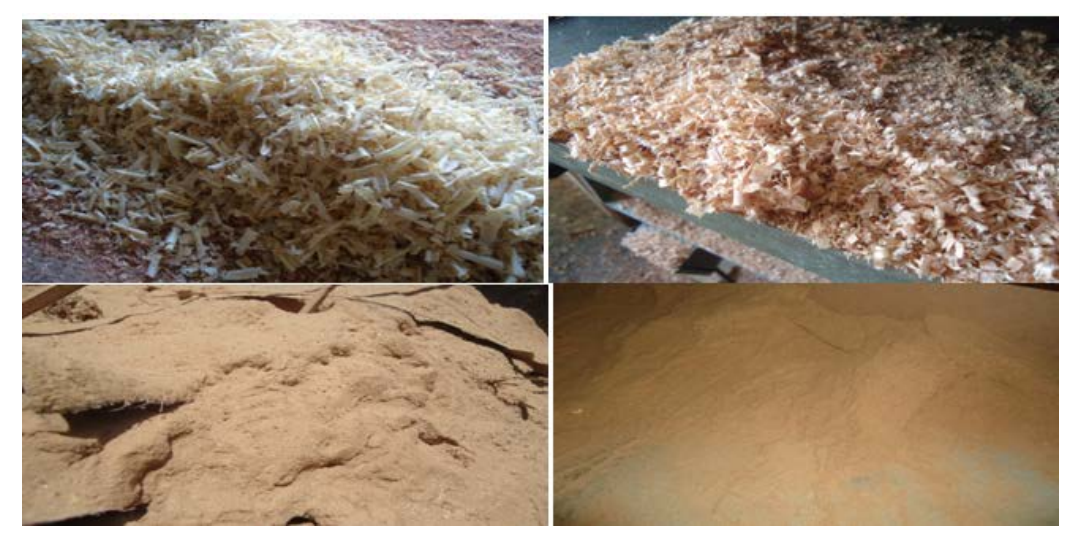

Figura 3: Catalogação da serragem e pó de serra de madeira, 2015.

Elaborado pelo autor, com base na pesquisa realizada. 


\section{DESENVOLVIMENTO}

A pesquisa foi desenvolvida em duas etapas, a primeira etapa com base na pesquisa de campo, registro fotográfico e referências bibliográficas para análise da atual situação do contexto geral das indústrias moveleiras situadas principalmente na região de Belo Horizonte e identificação; classificação; destino e aproveitamento dos resíduos sólidos de madeira gerados pelo setor moveleiro durante o processo produtivo. A segunda etapa, usando ferramentas do design, para gerar soluções de aproveitamento desses resíduos de madeira, através da pesquisa de materiais, tecnologias e testes para viabilizar a aplicação desse resíduo e potencializar como material de construção para novos produtos com visibilidade mercadológica para setor da decoração. Permitindo uma melhor compreensão do design como um instrumento fundamental para obter soluções que envolva descarte $x$ reciclagem a fim de minimizar os problemas ambientais.

O projeto seguiu diversas etapas diferenciadas, conforme a necessidade envolvida para o melhor aproveitamento da serragem e através de soluções visando à consciência ambiental por meio de um processo que busca o caminho que viabiliza uma contribuição com o desenvolvimento sustentável. Sendo assim, foram desenvolvidos vários modelos e testes de acordo com os fatores definidos pelo briefing.

Na etapa do briefing foi possível perceber que o setor da construção civil e decoração existem certas carências de produtos com qualidade e estética a partir de resíduos, e dentro desse setor os produtos mais desenvolvidos com uso de outros materiais residuais e o seu reaproveitamento foram de revestimentos de parede e piso. Os revestimentos de paredes têm por finalidade regularizar a superfície, aumentar a resistência da parede e proporcionar estética e acabamento. Os tipos de revestimentos de paredes são definidos a partir do material utilizado em revestimentos com argamassa e sem argamassa. Para isso, foi necessário estudar as etapas do processo de instalação de um revestimento comum adquirido por diversos usuários e analisar similares e correlatos.

O conceito do produto é um revestimento de parede para ambientes internos a partir da mistura do pó de MDF + aglutinante, com facilidade de aplicação através da cola de contato. Um produto decorativo, interativo e através do movimento da placa o usuário pode criar a composição que desejar em sua parede. Palavras - chaves do conceito: Movimente; Crie; Decore.

A partir da definição do conceito, as alternativas geradas seguiram os requisitos definidos e as três palavras chaves determinadas já apresentadas. Pensando na ideia de movimento, criação e decoração as soluções foram pensadas a fim de permitir a interação do cliente com o produto e valorizar a experiência do usuário durante o momento da aplicação. A geração de alternativas foi desenvolvida em duas fases. A fase 1 buscou explorar técnicas e formas de aplicação do produto. Já na fase 2, o produto foi pensando tanto na sua forma como na função do produto para priorizar a interação do usuário com o produto. Além dos desenhos em 2D, foram desenvolvidos mockups para criar uma maior percepção da forma e dimensão do produto e testes com aglutinantes para aplicar na alternativa escolhida (Figura 7). Essa evidência pontos importantes na definição dos requisitos e conceitos do projeto. Sendo assim, a alternativa escolhida atendeu a finalidade final do projeto de ser revestimento de 
parede para ambientes internos com possibilidade de o usuário criar várias composições no local de aplicação.

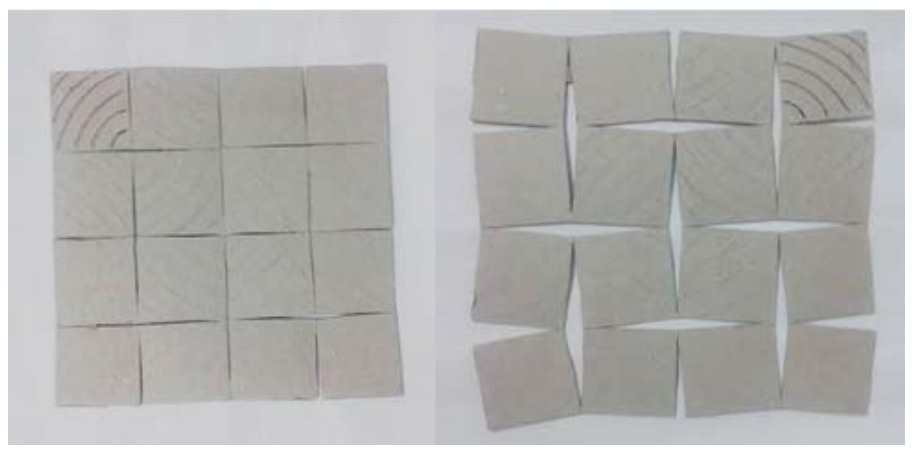

Figura 7. Alternativa Escolhida.

Elaborado pelo autor, com base na pesquisa realizada, 2015.

A fase 2 que incluiu os testes com aglutinantes foi de extrema importância para avaliar o potencial do material com diversos tipos de aglutinantes. $O$ teste priorizou utilizar aglutinantes não nocivos ao meio ambiente, mas também não foram descartados outros tipos de aglutinantes com propriedades eficazes com soluções a ponto de viabilizar a construção desse novo material. A alternativa escolhida foi a com os seguintes materiais: cola de amido + MDF em pó + silicone de vedação para um resultado final resistente com característica elástica, emborrachada e sem a produção de mofos e fungos. Resultado obtido depois da mistura e secagem da peça (Figura 8):

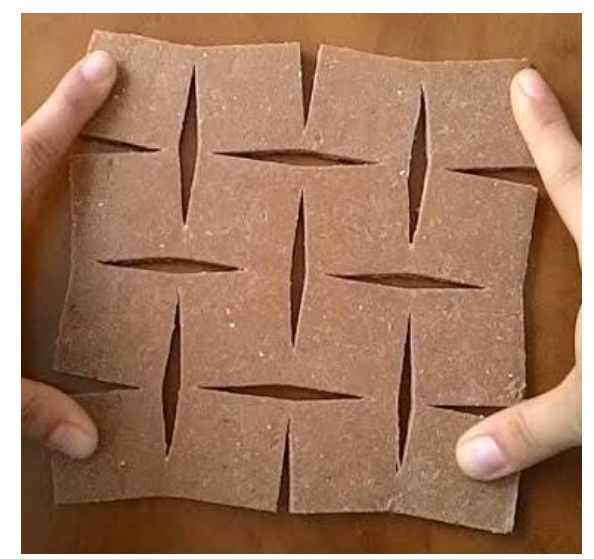

Figura 8: Resultado do teste utilizando silicone de vedação. Refinamento da Alternativa. Elaborado pelo autor, com base na pesquisa realizada, 2015.

a) Possibilidade de composição do revestimento para aplicação na parede (Figura 9): 

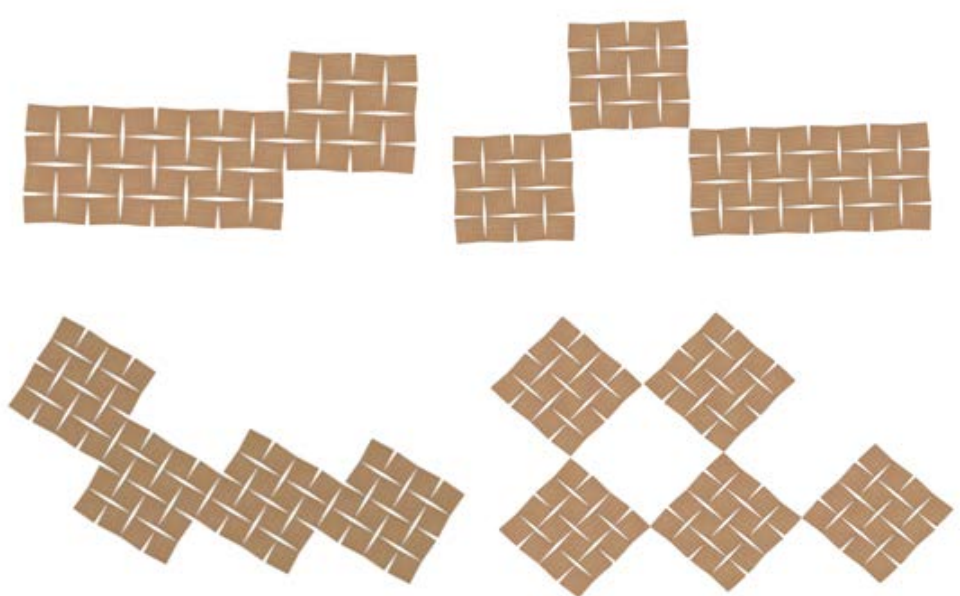

Figura 9: Possibilidades de Composição para Possíveis Aplicações do Revestimento na Parede. Elaborado pelo autor, com base na pesquisa realizada, 2015.

b) Tipos aplicação na superfície do Revestimento de parede para diferentes texturas (Figura 10):

\section{WALLTION}
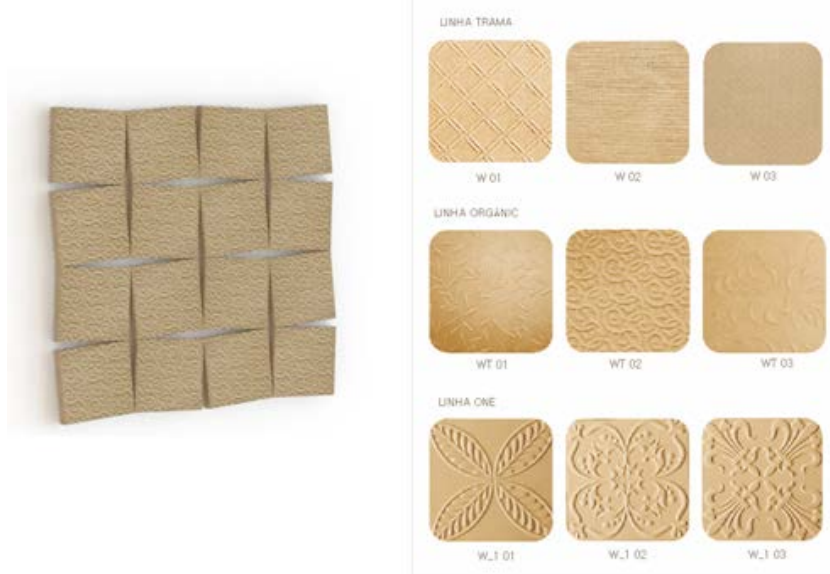

Figura 10: Linha de texturas do revestimento de parede.

Elaborado pelo autor, com base na pesquisa realizada, 2015.

Este projeto priorizou aspectos e padrões ergonômicos, entre eles seu peso e dimensões para fácil manuseio da peça dentro dos padrões convencionais de revestimentos de parede, resistência e não oferecendo riscos a saúde por não fazer uso de produtos tóxicos. Todos os atributos do projeto seguem discriminados abaixo:

i. Design: Ecologicamente viável, possui elementos estéticos expressivos com linhas retas para efeito criativo e moderno. Além de serem modulares e com facilidade de recorte na peça. Sistema inovador que permite o movimento da peça criando uma experiência e interação com o usuário podendo ser instalado como desejar, com diversas composições.

ii. Impactos ambientais: O produto visa minimizar os impactos causados pelos resíduos produzidos pelas empresas, contribuindo de forma satisfatória com a 
diminuição da poluição causadas devidos o acumulo do resíduo. Realização e contribuição com pesquisas e testes com aglutinantes biodegradáveis.

iii. Produção: Sem a necessidade de levar a peça para forno e sem uso de água durante o seu processo de fabricação. Através da mistura do Componente A + Componente $B$ prensados sob moldes de silicone com os rasgos já posicionados, a peça é retirada após sua cura de 6 horas. Com acabamentos simples para retirada de rebarbas da peça, depois separadas para venda.

iv. Economia: Contribui com o aproveitamento dos resíduos, aumentando o ciclo de vida da matéria-prima e minimizando o desgaste das reservas naturais. Além de ser economicamente viável, pois sua produção permite ser seriada por ser um produto modular e novamente reaproveitado após o consumo.

v. Mercado: O produto será comercializado a partir de um aplicativo com o nome do produto, nesse aplicativo a pessoa poderá conhecer o catalogo de texturas da peça e escolher, logo é apresentado o produto e as opções de alinhamento da peça, com a opção de ambientar com as dimensões reais da sua parede e cria a composição desejada. Ao fechar a encomenda são registras a quantidade de peças para o projeto e finalizada a venda. O produto chegará na casa das pessoas com manual e a imagem da composição escolhida para pessoa montar de acordo com a composição criada pelo aplicativo.

vi. Transporte: O aplicativo permitirá a comodidade do usuário de visualizar como o produto ficará em sua parede a partir da textura e composição definida por ele. Serão entregues na casa do cliente, as peças possui facilidade de transporte e instalação, devido o seu peso leve e com uma dimensão fácil de manusear.

\section{CONCLUSÃO}

O objetivo principal foi desenvolver um produto a partir de pesquisas e tecnologias além de contribuir com a conscientização das pessoas e empresas para o melhor aproveitamento do resíduo de MDF estudado no projeto. Na pesquisa foi possível estabelecer diversas propriedades e características do material aglutinado com alternativas biodegradáveis, contribuindo com a minimização dos impactos ambientais. Cabe ressaltar que o aspecto estético obtido através de um processo simples de fabricação do revestimento de parede ainda será necessário aperfeiçoamento da peça. É necessário avaliar sua resistência, flexibilidade, tipos de fixação, avaliar e pontuar de forma mais concisa o seu desempenho ambiental, possibilidade de coloração do revestimento e sua forma de comercialização, avaliar outras possibilidade de produção ou até mesmo de outro componente com o aglutinante mais ecológico e principalmente buscar por incentivos para o desenvolvimento de tecnologias para analisar a viabilidade de aplicação em escala industrial, além de explorar de forma mais aprofundada estudos e técnicas com os aglutinantes utilizados nos testes. Faz-se necessário o incentivo a pesquisa no meio acadêmico e convidar empresas a discutir práticas de reciclagem, de separação e catalogação dos seus resíduos para o uso mais racional dos recursos naturais além de explorar novas tecnologias e mercados. 


\section{REFERÊNCIAS}

BOM, Roberto Pedro. Cadeira de Painéis de Madeira. Processo Produtivo de Painéis MDF. Centro Universitário de União da Vitória. União da Vitória - PR, 2008.

CARVALHO, A. F. Disposição de resíduos sólidos na indústria de compósitos. Anais do III Seminário "Desenvolvimento Sustentável e a Reciclagem na Construção Civil: Práticas Recomendadas", 2000.

CÉSAR, Sandro Fábio; GUEDES, João Paulo Leite; CUNHA, Rita Dione Araujo. Aplicação de Design com Sustentabilidade na Produção de Móveis a partir de Madeira Reciclada. V Encontro Nacional e III Encontro Latino - Americano sobre Edificações e Comunidades Sustentáveis, Recife, 2009.

CUNHA, Rita Dione Araújo Cunha; CÉSAR, Sandro Fábio; GUEDES, João Paulo Leite. Aplicabilidade da madeira reciclada no design de móveis - conceito desenvolvido de mesas de centro. 9o Congresso Brasileiro de Pesquisa e Desenvolvimento em Design, 2010.

GRUPO SUSTENTAX. Mercado para Produtos Sustentáveis. Febraban. 29 de junho de 2010.

NAHUZ, Márcio. Resíduos da Indústria Moveleira . III Madetec. III Seminário de Produtos Sólidos de Madeira de Eucalipto e Tecnologias Emergentes para a Indústria Moveleira. São Paulo, 2005.

OLIVEIRA, Edmar Bonfim. Desenvolvimento Sustentável e produção Limpa: Estudo de um caso em uma empresa do setor moveleiro. Porto Alegre, 2009.

RODRIGUES, Lia; CASTILLO, Leonardo. A sustentabilidade e o design de mobiliário: um entrelace histórico. 9 Congresso Brasileiro de Pesquisa e Desenvolvimento em Design, 2010.

SOUZA, Marcos Martins. Desenvolvimento de Produtos a Partir de Resíduos de Madeiras: A Importância do Design na Sustentabilidade do Setor Moveleiro. Instituto de Estudo Superior da Amazônia. Curso de Bacharelado em Design Projeto Produto. Belém, 2008.

TEIXEIRA, Marcelo Geraldo; CÉSAR, Sandro Fábio. Produção de Compósito com Resíduo de Madeira no Contexto da Ecologia Industrial. Anais do 10 Encontro Brasileiro em Madeiras e em Estruturas de Madeira - EBRAMEM, São Paulo - SP, 2006.

TUOTO, Marco. Levantamento sobre geração de resíduos provenientes da atividade madeireira e proposição de diretrizes para políticas, normas e condutas técnicas para promover o seu uso adequado. Ministério do meio ambiente. Secretária de Mudanças Climáticas e Qualidade Ambiental. Secretaria de Biodiversidade e Florestas Projeto PNUD BRA 00/20. Curitiba-PR, 2009. 